\title{
System architecture to select the charging station by optimizing the travel time considering the destination of electric vehicle drivers in smart cities
}

\author{
Ibrahim El-Fedany ${ }^{1}$, Driss Kiouach ${ }^{2}$, Rachid Alaoui ${ }^{3}$ \\ ${ }^{1,2}$ Modeling, Systems and Technologies of Information Team, High School of Technology, Ibn Zohr University, \\ Agadir, Morocco \\ ${ }^{3}$ LRIT Laboratory, Faculty of Sciences, Mohammed V University, Rabat, Morocco \\ ${ }^{3}$ LASTIMI Laboratory, Higher School of Technology-Sales, Mohammed V University, Rabat, Morocco
}

\begin{tabular}{|c|c|}
\hline Article Info & ABSTRACT \\
\hline Article history: & \multirow{10}{*}{$\begin{array}{l}\text { The main limitations of electric vehicles are the limited scope of the battery } \\
\text { and their relatively long charging times. This may cause discomfort to } \\
\text { drivers of electric vehicles due to a long waiting period at the service } \\
\text { of the charging station, during their trips. In this paper, we suggest a model } \\
\text { system based on argorithms, allowing the management of charging plans } \\
\text { of electric vehicles to travel on the road to their destination in order to } \\
\text { minimize the duration of the drivers' journey. The proposed system decision } \\
\text { to select the charging station, during advance reservation of electric vehicles, } \\
\text { take into account the time of arrival of electric vehicles at charging stations, } \\
\text { the expected charging time at charging stations, the local status } \\
\text { of the charging stations in real time, and the amount of energy sufficient for } \\
\text { the electric vehicle to arrive at the selected charging station. Furthermore, } \\
\text { the system periodically updates the electric vehicule reservations to adjust } \\
\text { their recharge plans, when they reach their selected earlier station compared } \\
\text { to other vehicules requesting new reservations, or they may not arrive as they } \\
\text { were forecast, due to traffic jams on the road or certain reluctance on the part } \\
\text { of the driver. }\end{array}$} \\
\hline Received Mar 9, 2019 & \\
\hline Revised Sep 26, 2019 & \\
\hline Accepted Nov 30, 2019 & \\
\hline Keywords: & \\
\hline Charging reservation & \\
\hline Driver journey duration & \\
\hline Electric vehicle & \\
\hline Public charging station & \\
\hline Smart city & \\
\hline
\end{tabular}

This is an open access article under the $\underline{C C B Y-S A}$ license.

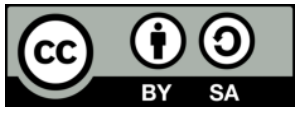

Corresponding Author:

Ibrahim El-Fedany,

Modeling, Systems and Technologies of Information Team,

High School of Technology, Ibn Zohr University, Agadir 80000, Morocco.

Email: fedany82@gmail.com

\section{INTRODUCTION}

Research institutes, government agencies and industries worldwide are increasingly interested in improving urban life in cities of tomorrow smart cities, while reducing the impact on the environment. The replacement of internal combustion engine (ICE) vehicles which are responsible for a decreasing share of pollutants [1] by electric vehicles (EVs) would reduce the accumulation of pollutants on the road and the dependence on fossil fuels Also, they improve the local air quality index (AQI) by 13 points and the health of citizens [2] and many countries have provided important guidelines for adopting electric vehicles $[3,4]$. Therefore, electric vehicles are considered one of the main components of transport systems in smart cities. Likewise, many intelligent city are programing to electrify their transport systems; so the number of EVs is expected to increase significantly in the near future [5]. But, the ability of battery is one of the main factors preventing wide adoption of EVs [6]. This ability or distance that the vehicle can cover before it needs to be recharged is among the main factors preventing wide adoption of EVs [6]. 
Generally, the range for a modern EV is between $100 \mathrm{~km}$ and $500 \mathrm{~km}$. When EVs travel during journeys, they must be recharged at the electric charging stations. Each CS is equipped with a few charging sockets and according to the current charging technology, the EV charging time can normally exceed 30 minutes [7 -9]. Moreover, if all CS sockets charging are occupied, any EV incoming must wait until one of the sockets charging will available. It is important for an EV to reduce its waiting time to select the CSS that is not too occupied.

On the other hand, EVs drivers have their individual journeys, they may not be ready to wait long enough to charge their electric vehicles. The selection of a far CS from the trip destination is no longer desirable because they prefer to reach their travel destinations as soon as possible. Therefore, the manner to manage EV' charging processes, to improve their drivers comfort, is a critical research issue for the long-term success of the EV industry and the smart cities' development to have eco-sustainable urban environment.

In order to minimize the average waiting time, the author in $[10,11]$ are proposed an EV itinerary planning algorithmsthe and a distributed strategy based on a combination of algorithms that ensure the consistent use of EV charging demand along the highway proposed in [12]. EVs will pass through all CSs; they should report their charging reservation. Hence, to prove the effectiveness of these algorithms, the authors adopt certain hypotheses, for example, each station has an equalcharge capacity. The uses of all the stations must be equal so that the waiting time is minimized. A scheduling algorithm based on the Dijkstras algorithm is proposed [13] to find the optimal path for EVs and ameliorate the network performance and transport systems, such as reducing the waiting time on the EV side and the charge balance between several CSs. Accordingly, to evaluate their algorithm, it is assumed that the driver of the EV will choose the nearest station when the battery level is less than 30\%. In [14], a pricing-sensitive system is implemented to optimize online recharge planning. In [15], the authors propose a communication framework based on the use of roadside units to link the information flow from CS to EV. CSs publish their own status information, including the waiting time, location, price, and abilities to EVs as information subscribers. The CS selection is based on the minimum charging waiting time. In [16], they built an EV charging system as used in [15], in order to increase data security while reducing information access times. The proposed scheme can capture and predict CS status with more precision.

The work in [17] presents an exploitation of the distributed energy resources in order to minimize the EV charging time while optimizing the efficiency of the electrical infrastructure. Thus, the results of comparing the CS selection schemes which are based on the nearest distance and the minimum waiting time show that the latter works better in the city' scenario whose high density of EVs. In [1 8], the others propose a centralized system model that allows the EVs charging' management, with a view to minimize the duration of the drivers' journeys due to an intermediate charging EV at the CSs. The EV which has been informed by the CS selected for their charging plan, declares its reservation (including its time of arrival and the expected charging time at this CS) to the GA. Regarding the uncertainty linked to traffic due to traffic jams, a periodic update of reservations is also carried out to adjust the charge plans of the VEs [19]. In [20], the authors implement an architecture of a load planning system for all the EVs as a function of the queue time and without taking into account the other EVs that travelling towards the same CS recommended.The work in [21] offers a centralized reservation service in order to select a CS that will not be very congested to improve the driver's comfort by minimizing the waiting time to charge their batteries. They jointly consider the battery change procedure used locally in the CS as already has been taken by [22]. That means each CS is able to maintain a number of batteries for switching. Therefore, EVs must use the same standard battery as those in the CSs. In [23], the authors proposed a static system that presents a charging recommendation for an electric vehicle driver, the latter can not obtain any information on the CS, that is to say that the CS works or not, how many vehicles are waiting for charging and what charging points are available.

Most of the previous work [13-23] focuses on the city scenario. The EV goes to a single geographically distributed CS for charging. Thus, the selection of CS by an EV takes into account the EVs' reservations in a Global Aggregator (GA). The latter manages the EVs cha rging requests to optimize the estimation of their travel time including the charging waiting time at the CS. This operation is executed when GA receives the VEs' charging requests and the decision taken by the GA supose that the charging stations operates with "the first come is the first served" policy (FCFS). Consequently, the waiting time as well as the estimated trip' time for reservations previously confirmed by the EVs in a CS have no value if a new reservation of an EV perform in the same CS; it will arrive earlier than others. Similarly, the same effect on the decision of the CS selection made by the GA if one or more EVs will not have arrived at the CSs to follow their reservation request. Besides, the decision proposed by the GA to the selection of a CS following a reservation request from EV on a trip, it doesn't matter if the current power of EVs battery is insufficient to reach the selected CS. 
In this context, the interest of our work presented in this paper is to set up a model system allowing the optimization of charging reservation requests of the VEs that moves towards their destinations. This system consists of the algorithms which will be executed at CS and AG, when the latter receives a charging request by EV. Thus, the decision of selection of CS given by the GA take into consideration the amount of sufficient energy so that the EV would arrive at the selected CS, the elimination of EVs reservations whose expected arrival time exceed the current time, and the CS change notification for EVs moving to its destination; due to a new reservation of an EV that will arrive before the EVs having a reservation in the same CS. Here, $3 \mathrm{G} / \mathrm{LTE}$ is applied so as not to be delayed, thanks to ubiquitous communication. The rest of this paper is organized as follows. Section II, the design of the model system proposed. In Section III, the algorithm that constitutes the model system. Section IV, we presents the results and discussion. Finally, the conclusion of document in section V.

\section{PROPOSED SYSTEM MODEL}

\subsection{Assumptions}

In this paper, we assume that all CSs are geographically deployed in a smart city, EVs are equipped with a global position system GPS [24, 25], and wireless communication devices such as $4 \mathrm{G}$ are available. This allows them to determine their current position and to communicate with a centralized entity, Global Aggregate (GA), which manages the request/response services. The latter are called by all the VEs to take the recharge reservation decision. Each CS has multiple charging slots so that a number of EVs could be loaded in parallel. In order to plan the EV charging queue in the CS, this latter adopts the first-come-first-served (FCFS) policy. This means that parking an EV with an early arrival time will be scheduled with a higher charging priority. If an SC is fully occupied (namely, all of its charging loca tions are currently in use), the incoming EVs must wait for one of its charging locations to be free. It is assumed that the number of places for waiting in the CS is unlimited; the EV can leave the CS before being fully charged. The calculation of the arrival time of an EV to a CS or to their destination is based on an initial maximum acceleration rate. Figure 1 shows EV charging transition states:

- Driving state: EVs move in the city where they are traveling to their destinations.

- Low battery Level state: the state where the EV system indicates the low battery level.

- Driver decision states: Here, the drivers are traveling to their destination, in order to optimize their journey time; they decide to plan the battery charging of their EV

- Reservation state: the state at which the EV requests a charge reservation, or when it receives a notification coming from the GA in order to change the old reservation by proposing the new charging station, this notification is the result of execution the algorithm 3 by GA which provides a change of status to the CS selected.

- Moving to charging state: the state where the EV' driver confirms the reservation and moves to its selected CS. At the time of the trip, the EV may receive an indication to update the charging plan sent by the GA.

- Waiting line state: Upon the arrival of the EV to the selected CS and according to "the first-come, first-served FCFS policy", he will wait to be charged. It may be that the EV quits the queue for an unexpected reason, if there is enough energy, and he will resume his journey of destination by requesting another plan of reservation charge.

- Charging state: The EV is charging and will switch to driving once its battery is fully or partially charged.



Figure 1. Flowchart of EV charging transition state 


\subsection{The system communication scenario}

The proposed system consists of three main components the EVs that request the charge reservation, the CSs that locally manages the queuing of EVs by applying the CFCS policy, and a centralized server GA that manages the reservation requests of the EVs based on the execution of the algorithm 3 and sends them the information concerning the CS selected such as, position, expected charging time etc. In addition each CS executes, following a request sent by the GA to each new request for reservation of $\mathrm{EV}$, the algorithm 1 to obtain the list containing the queue of their charge sockets, this list will be sent to the GA. The message flow between these three components is described as follows, Figure 2:

- Step 1: The EV is in moving and in a certain time the EV' driver wants to plan the charge of their EV along the path of their destination, or when the level of their EV is low. It sends a CS selection request to the GA.

- Step 2: After the receipt of the reservation request of an EV by the GA, the latter sends a request to all the CSs located in the same geographical area of the EV in order to send them their current queue.

- Step 3: Each CS that receives the request from GA, it runs the algorithm 1 in order to get the list that contains the wait time of each charging socket in CS, this list will be send to GA.

- Step 4: When the GA receives the list containing the current status of each CS, it executes the algorithm 3 which cancels the expired reservations and updates the list of reservations, after it sends the parameters of the CS selected to the EV requesting the reservation.

- Step 5: The driver of the EV confirms the proposal sent by the GA and he moves to his destination by following the route that provides for the recharge of his EV. When the GA receives acceptance of the EV to the selected CS, it places it in the reservation requesting list and it sends a notification to the EVs which have an update of their charge plans.

- Step 6: When the EV enters in CS, it will take the queue if there is not a free charge socket in CS whose CFCS policy is applied. The CS informs the GA at the arrival of the EV to eliminate it from the reservation list.

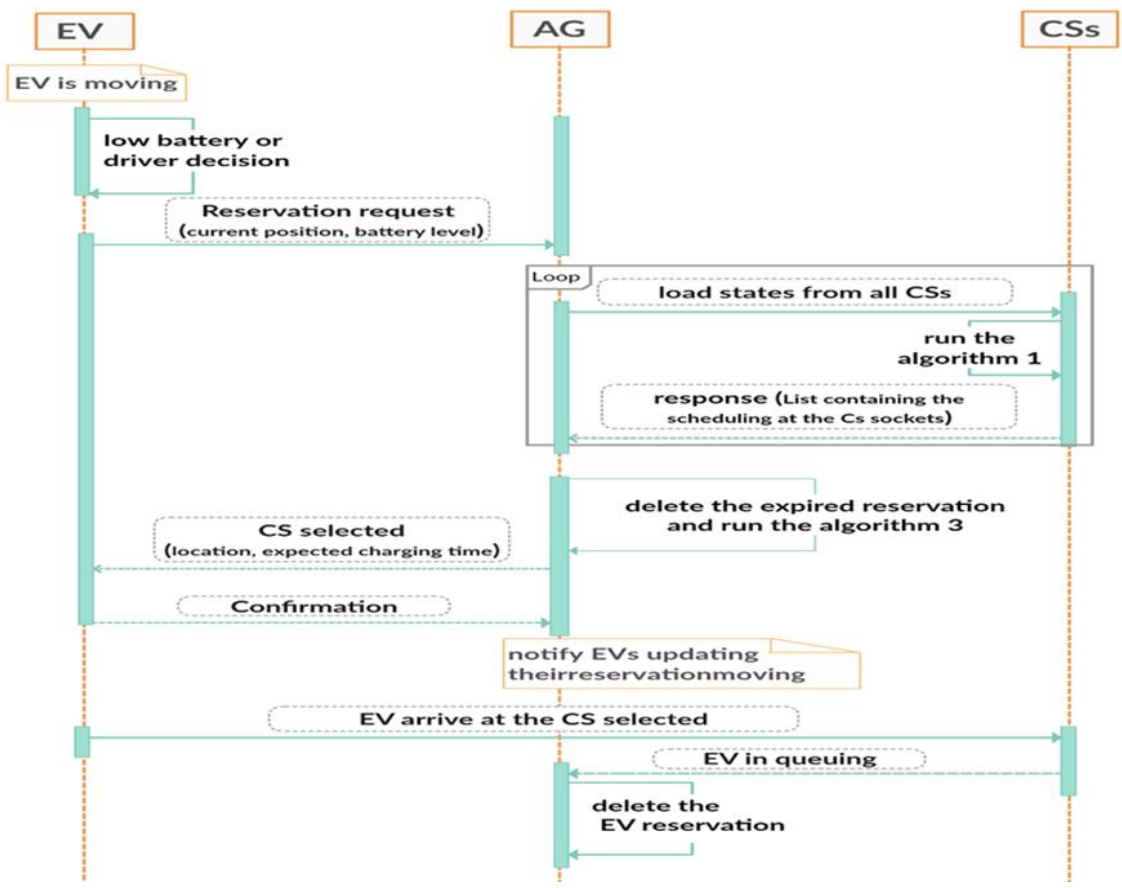

Figure 2. Communication scenario between EV, GA, and CSs

\section{SYSTEM ALGORITHM}

The new EVr charge reservation request can have a negative effect on previous reservations, especially reservations in the CS where the EVr will have it, (see algorithms in [17-22]). Assuming that in a CS there are EVs that are confirmed their charge reservations and that the GA affects to the same CS a new reservation of another EVr. The charging time estimated by the GA during the selection of CS takes into account the arrival time and the charging time of the previous reservations, it is like an external queue for 
the reservation. Thus, the time of estimated charge for previous reservations of EVs in a CS will not be the same; if there is a new EV reservation at the same CS. it will arrive rather than the other previous. In addition, think about what one gets as estimated travel time to the next reservation request, if one or more EVs that have no longer arrived at their CS reservation due to an unexpected change in the EV driver's plan or the current battery power are insufficient for the EV to a rrive at the selected CS. In order to answer the points presented above, our contribution proposes a system model consists of three algorithms, the first one is executed with the CS and the two others are triggered, with each new request for reservation of an EV, by GA. The main task of each algorithm is:

- Algorithm 1 returns a list containing an estimate of the valid charging time for each socket charge of CS.

- Algorithm 2 is an intermediate algorithm used in the third algorithm that returns a TLIST list, which registers for each EV a CS selected charge based on the optimization of the trip time and takes into account the a mount of enough energy for the EV to arrive at the CS.

- The algorithm 3 is responsible for optimizing the reservations of the EVs, it browse all the EVs having previous reservations to the purpose of notifying the EVs where their estimated charging times suffered a change due of the new reservation, using the algorithm 2 at each iteration to return the list TLIST which will be sorted in descending order according to the EV arrival time to its selected CS. The Table 1 contains the meaning of the variables used in the all system algorithms.

Table 1. List of nomenclatures

\begin{tabular}{|c|c|}
\hline Abbreviation & Full Meaning \\
\hline LIST & Output including available time per charging slot at CS \\
\hline$N_{\text {slot }}$ & Number of charging slot at CS \\
\hline$T_{\text {cur }}$ & Current time in network \\
\hline$N_{e v}$ & Number of EVs in CS \\
\hline$\beta$ & Charging power at CS \\
\hline$E_{e v_{j}}^{\max }$ & Full volume of EV battery \\
\hline$E_{e v_{j}}^{c u r}$ & Current volume of EV battery \\
\hline$T_{e v_{i}}^{f i n}$ & Charging finish time of EV \\
\hline LR & $\begin{array}{l}\text { List, in the form of dictionary, contain s in each line an EVs requesting a recharge reservation with their selected station } \\
\text { and the time of arrival to the latter. }\end{array}$ \\
\hline TLIST & Temporary list of EVs with best choice of CS \\
\hline CSLIST & list including LISTs of each CS \\
\hline $\mathrm{T}_{\min T r i p}$ & The minimum time for EV arrives at their des tination \\
\hline$C S_{j_{\min }}$ & The CS selected for an EV to have the minimum travel time \\
\hline$N_{C S}$ & Number of CS in smart city \\
\hline$T_{E V_{i} \rightarrow C S_{i}}^{a r r}$ & The EV's arrival time of index $i$ to a CS of index $j$. \\
\hline$T_{E V_{i} \rightarrow C S_{j}}^{\operatorname{tran}}$ & The EV's travelling time of index i to reach CS of index j. \\
\hline$T_{E V_{i} \rightarrow C S_{i}}^{c h a r}$ & Expected charging time upon arrival of $E V_{i}$ \\
\hline$T_{C S_{j} \rightarrow T R_{E V_{i}}}^{\min }$ & Minimum travelling time from a $C S_{j}$ to $E V_{i}$ 's trip destination \\
\hline$E_{E V_{i} \rightarrow C S_{i}}^{\operatorname{tran}}$ & Estimation of the energy consumed for moving the $\mathrm{EVi}$ to the $\mathrm{CSj}$ \\
\hline $\mathrm{S}_{\mathrm{EVi}}$ & Moving speed of EV \\
\hline$\alpha$ & Electric energy consumed per meter \\
\hline
\end{tabular}

\subsection{Estimating the available times for charging at charging station: Algorithm 1}

For the VEs to make a charge reservation in one of the charging stations deployed in a smart city, first of all, it is necessary to estimate the time available for each charging slot, depending on the knowledge of the number and the Battery status of the EVs that are currently in these CSs. As shown in lines 1,2 and 3 of algorithm 1, the current time in the network, as noted by Tcur, is estimated as the available charging tim $\mathrm{e}$ for each location charge of a CS, where their socket number is chara cterized by Ns, if no EV is in the queue. In this case, the list containing a number of Tcur is returned directly. That means that the CS charging locations are currently available. While, if the CS queue contains an EV number noted by Nev, line 4 , the loop operation starts from sorting the Nev queue. Taking into account the procedure of parallel charge via several locations charging and the FCFS policy, each iteration of the loop, from line 5 to 10 , the duration for each $\mathrm{EVj}$ in the queue to be fully recharged is given by $\frac{E_{e v_{j}}^{\max }-E_{e v_{j}}^{c u r}}{\beta}$. The latter will be added to the first available time at the top of the list, as indicated by LIST.GET (0), by obtaining the loading end time $T_{e v_{j}}^{f i n}$ of each $\mathrm{EVj}$, line 7 . In line 8 , the first time available, LIST GET (0), will be replaced by $T_{e v_{j}}^{f i n}$. So that the shortest time of the charge obtained by LIST GET (0) is used in each iteration line 9. The list will be sorted in ascending order and the above loop operation will end when all EVj were processed, then LIST is returned to line 12 . 
Algorithm 1: Charging time estimated at charging station

1: $\quad$ for $(\mathrm{i}=1 ; \mathrm{I}<=$ TLIST.getSize ()$; \mathrm{i}++)$ do

2: $\quad C S_{j_{\text {min }}}=C S_{\mathrm{k}}$, such as $T_{E V_{i} \rightarrow C S_{j_{\min }}}^{a r r}=\arg _{k<N_{C S}} \min \left(T_{E V_{i} \rightarrow C S_{k}}^{a r r}\right)$

3: $\quad$ Calculate $T_{i, k}^{\text {Trip }}$ according to (1)

4: $\quad T^{\text {minTrip }}=T_{i, k}^{\text {Trip }}$

5: $\quad$ for $\left(\mathrm{j}=1 ; \mathrm{j} \leq N_{C S} ; \mathrm{j}++\right)$ do

6: $\quad$ if $\left(E_{E V_{i}}^{\text {curr }} \geq E_{E V_{i} \rightarrow C S_{j}}^{\text {tran }}\right)$ then

7: $\quad$ Calculate $T_{i, j}^{\text {Trip }}$ according to (1)

8: $\quad$ if $\left(T_{i, j}^{\text {Trip }}<T^{\text {minTrip }}\right)$ then

9: $\quad T^{\text {minTrip }}=T_{i, j}^{\text {Trip }}$

10 :

11:

12:

13:

14:

15:

16 :

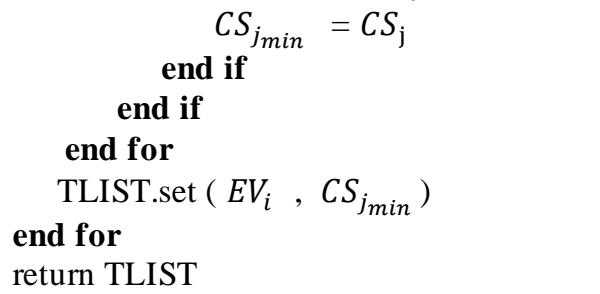

\subsection{Reassignment of $\mathrm{CS}$ for each $\mathrm{EV}$ had a reservation in GA: Algorithm 2}

This algorithm makes it possible to affect each EVi in the TLIST list, dictionary type with EV represents the key and CS its value, at a CSj where the travel time $T_{i, j}^{\text {Trip }}$ of the EVi toward its destination, including the charging time in the $\mathrm{CSj}$, will be the minimum, the loop of the line 1 to 15 .



The calculation of $T_{i, j}^{\text {Trip }}$ is defined as follows:

$$
\begin{aligned}
& \text { if (CSLIST(j).get(0) > } T_{E V_{i} \rightarrow C S_{j}}^{a r r} \text { ) then } \\
& \qquad T_{i, j}^{\text {Trip }}=\operatorname{CSLIST}(\mathrm{j}) \cdot \operatorname{get}(0)+T_{E V_{i} \rightarrow C S_{j}}^{c h a r}+T_{C S_{j} \rightarrow T R_{E V_{i}}}^{\min } \\
& \text { else } \\
& \qquad T_{i, j}^{\text {Trip }}=T_{E V_{i} \rightarrow C S_{j}}^{a r r}+T_{E V_{i} \rightarrow C S_{j}}^{c h a r}+T_{C S_{j} \rightarrow T R_{E V_{i}}}^{\min } \\
& \text { end if }
\end{aligned}
$$

Here, CSLIST (j) .get (0) represents the first available time at the top of the list LIST, LIST.GET (0), of $\mathrm{j}^{\text {th }} \mathrm{CS}\left(\mathrm{CS}_{\mathrm{j}}\right), T_{C S_{j} \rightarrow T R_{E V_{i}}}^{\min }$ is an estimate of the minimum travel time between the selected CS and the travel destination of the EVi , thus $T_{E V_{i} \rightarrow C S_{j}}^{c h a r}$ given by: 


$$
T_{E V_{i} \rightarrow C S_{j}}^{c h a r}=\frac{E_{e v j_{j}}^{\max }-E_{e v_{j}}^{c u r}+E_{E V_{i} \rightarrow C S_{j}}^{\operatorname{tran}}}{\beta} \text { with } E_{E V_{i} \rightarrow C S_{j}}^{\operatorname{tran}}=\left(\mathrm{S}_{\mathrm{EVi}} \mathrm{x} T_{E V_{i} \rightarrow C S_{j}}^{\operatorname{tran}} \mathrm{x} \alpha\right) .
$$

Before going through all the CSs, from line 4 to 16 , with $N_{C S}$ representing the total number of CSs, we initialize $C S_{j_{\min }}$ by the nearest $C S_{k}$ based on the minimal arrival time $T_{E V_{i} \rightarrow C S_{k}}^{a r r}$ given by $\left(T_{c u r}+T_{E V_{i} \rightarrow C S_{k}}^{\operatorname{tran}}\right.$ ), between line 2 to 4 , and $T^{\text {minTrip }}$ by $T_{i, k}^{\text {Trip }}$. The loop which is between line 5 and 13 permits to iterate all CSs. at each iteration, the trip time computation $T_{i, j}^{\text {Trip }}$ can only be performed if the current quantity of energy in the EVi battery is greater than that which will be consumed between the EVi and CSj, line 5. This clause eliminates the selection of CSj that the EVi does not have enough energy to reach it. The $C S_{j_{\min }}$ obtained at the end of the iteration is affected to the EVi in the TLIST list, the line 14. Finally, we obtain the TLIST list of which each EV has a CS by which their travel time to its destination is the minimum, this list is sent to line 16 .

\subsection{The CS selection to EV with the optimal trip duration: Algorithm 3}

The arrival of an EVr charge reservation request to the GA triggers the execution of this algorithm; the latter starts the search for CS at which the travel time of the EVr towards its destination is the minimum, by updating the position parameters of each EV having a previous reservation recorded in the reservation list LR, line 1.

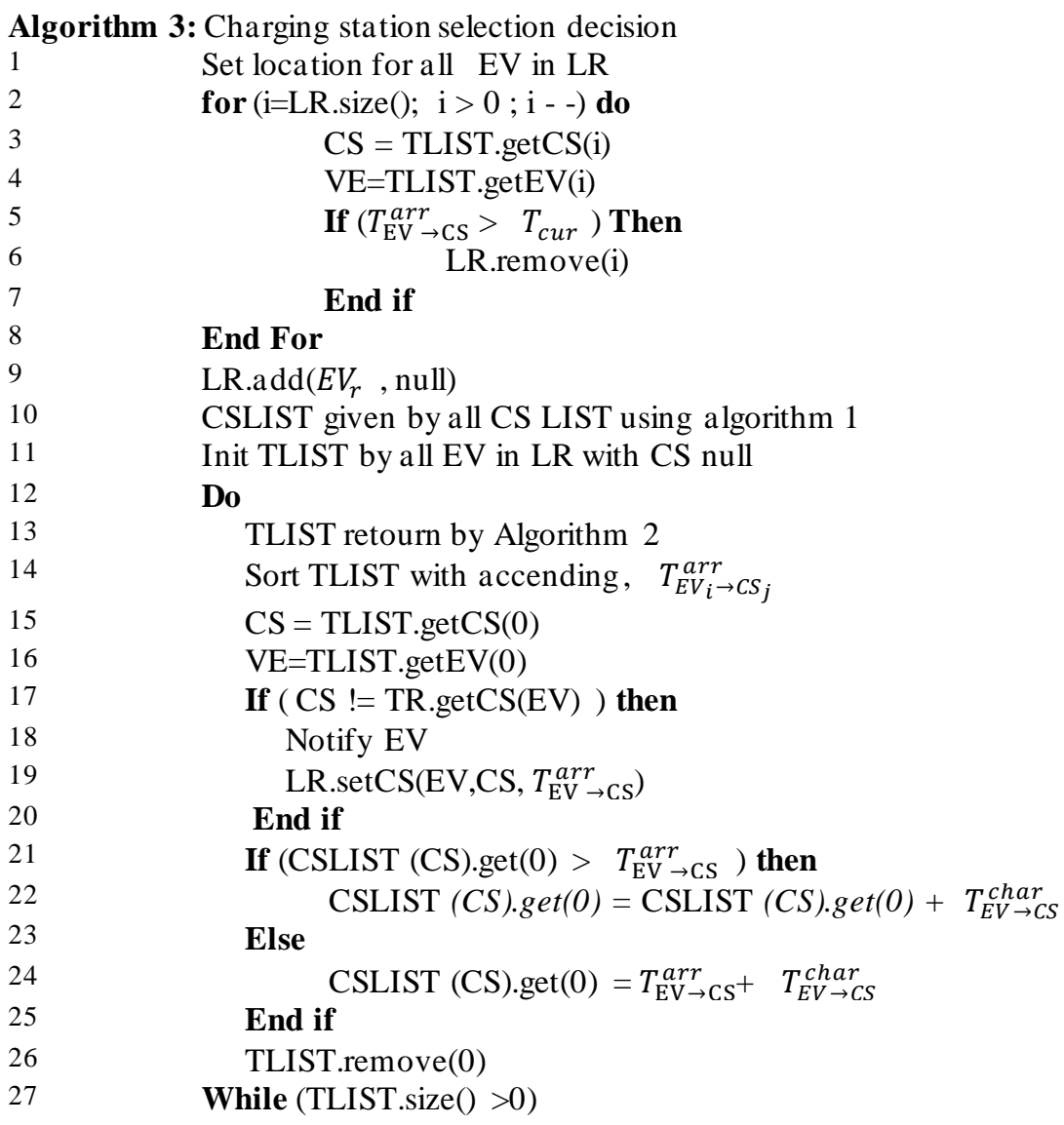

From line 2 to 8 , a loop to iterate the entire reservation list whose algorithm removes all reservations of EVs that their pre-estimated arrival time exceeds the current time, line 5. The addition of EV request being processed to the LR list, line 9. In the line 10, a call from the GA to all the CSs to send them their LIST list of the queue to update by running the algorithm 1 . These lists are registered and indexed by their CSs in the CSLIST list. The initialization of the intermediate list TLIST in line 11, by all the EVs of the list LR including last EVr so that all the reservations are canceled, with the aim of reserving a gain.

The execution of the algorithm 2, line 13, permit to fill the list TLIST by assigning for each EV the suitable CS, after this list will be sorted in increasing order in the line 14 based on the arrival time of each EV to their CS selection. Here, the first EV that will arrive at the CS of their optimal reservation is at the top of TLIST, as indicated by TLIST.getCS (0), the CS obtained will be compared by the CS registered in the LR 
list of the same EV and if they are different, a notification is sent to the EV so the new CS replaces the old in LR, from line 17 to 20 .

To estimate when a load slot will be available to load at EV arrival, we designate CSLIST (CS). Get (0) as the earliest available charge time in the LIST list of the CS selected and if $T_{\mathrm{EV} \rightarrow \mathrm{CS}}^{a r r}$ is earlier than the first available time to charge, CSLIST (CS) .get (0), the end charging time of EV is calculated by (CSLIST (CS).get $\left.(0)+T_{E V_{i} \rightarrow C S_{j}}^{c h a r}\right)$ or $\left(T_{\mathrm{EV} \rightarrow C S}^{a r r}+T_{E V \rightarrow C S}^{c h a r}\right)$ with $T_{E V \rightarrow C S}^{c h a r}$ is calculated according to (1), line 22 and 24. In line 26, the EV that is processed before will be removed from TLIST and the same treatment will be repeated so that all EVs update their CS selection charge including the last EVr, from line 12 to 27.

\section{RESULTS AND DISCUSSION}

\subsection{Configuration simulation}

We built a custom discrete event simulator in Java to evaluate the algorithms of the proposed system and compare them with the algorithms of previous work. As a case study, we simulated a network of electric vehicle drivers in an area of $8000 \times 18000 \mathrm{~m}^{2}$, which presented as the center of Agadir in Morocco. We obtained the charging stations locations in the region from [26]. As shown in Figure 3, seven fast charging stations are assumed, each containing ten charging slots with a fast charge rate of $80 \mathrm{KW}$.

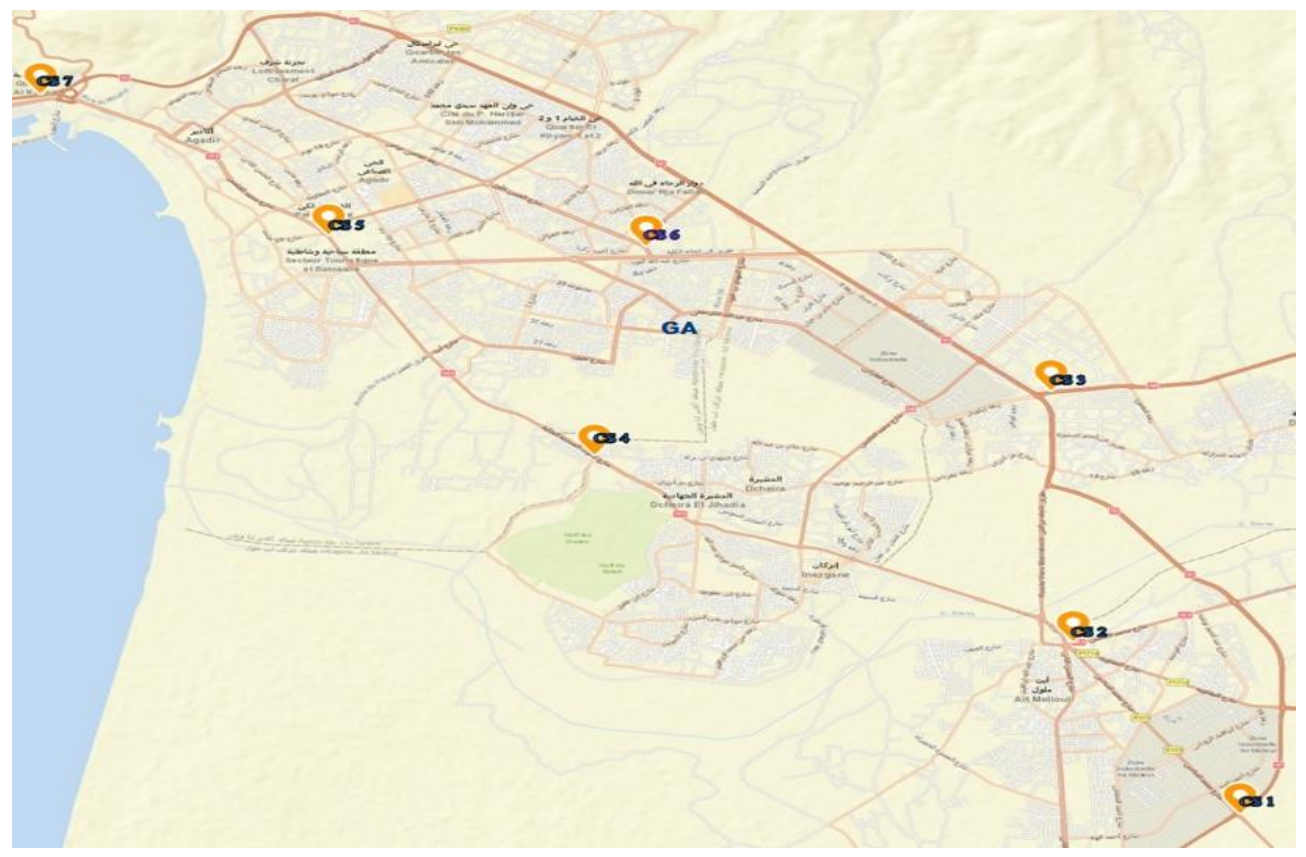

Figure 3. Simulation scenario of Agadir city

In addition, we assume that our simulation city has 1000 electric vehicles including 100 electric vehicles considered as comparable study samples. Thus, each EV entering the system follows a load specification according to a uniform distribution with maximum values such as, $100 \mathrm{KW}$ h for the maxim um capacity of electricity, $10 \mathrm{KW} \mathrm{h/100} \mathrm{Km} \mathrm{for} \mathrm{average} \mathrm{consumption,} 10 \%$ for the Status of Charge and $16 \mathrm{~m} / \mathrm{s}$ for maximum movement speed. In order to demonstrate the efficiency of our proposed algorithms (ALs 1), we compare them with the algorithms (ALs 2) proposed in [18] and which are extended in [19] as the benchmark performance. In the simulation experiments, we dispose two global aggregator (GA) in the city center in Agadir, the first one (GA 1) uses ALs 1 and the second one (GA 2) based on ALs 2, to manage the reservation charging requests of EVs. Thus, each EV traveling to its destination and needs to charging their battery, it sends the charge reservation request simultaneously to GA 1 and GA 2 . The results obtained after the execution of 100 times of the system, and the evaluation metrics are as follows:

- The average number of EVs that have trip duration including the charge time given by GA 1 equal to what given by GA 2, it evaluated as EV-Eq.

- The average number of EVs that have trip duration including the charge time given by ALs 1 lower than what given by ALs 2, it evaluated as TDA1LA2. 
- The avera ge number of EVs that have trip duration including the charge time given by ALs 1 greater than what given by ALs 2, it evaluated as TDA1UA2.

- The a verage trip duration of the EVs constituting the samples obtained by each algorithm in a moment ti where there is a new reservation request. AvgTDALs1 and AvgTDALs2 respectively represent the results obtained by ALs 1, our proposition, and ALs 2.

\subsection{Evaluation results}

Figure 4 shows a comparison between the prediction of ALs 1 and ALs 2 as a function of DeltaTi time which represents the interval between consecutive reservation requests of EVs. These are fixed on 400 EVs and the remarks drawn from this comparison are:

- The number of EVs, with a travel time predicted by ALs 1 less than that predicted by ALs 2, exceeds $60 \%$ whatever the Delta Ti time.

- The reduction in DeltaTi time results in an increase in the number of EVs with a travel time predicted by ALs 1 less than that predicted by ALs 2.

We can say that the algorithm 1 is more efficient compared to the algorithm 2 and especially in the peak period during which the charging reservation requests are the strongest. Figure 5 illustrates comparisons between the two algorithms as a function of the number of EVs that request the reservation. The latter is performed consecutively in each time interval deltaT which is fixed at 500 seconds and the remarks drawn from this comparison are:

- The number of EVs, with travel time is estimated by ALs 1 lower than that estimated by ALs 2, exceeds double.

- The increase in the number of electric vehicles requesting the reservation leads to an increase in the number of electric vehicles whose travel time provided by ALs 1 is lower than that provided by ALs 2 .

It can be concluded that our algorithm is effective in large smart cities that have a larger number of EVs.

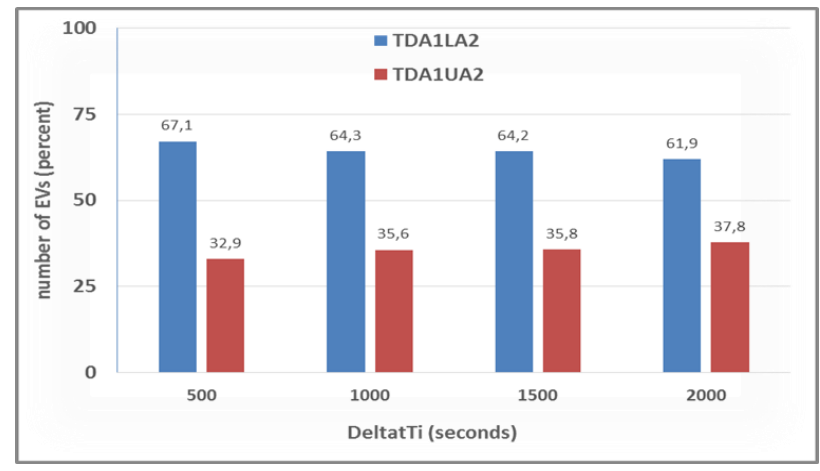

Figure 4. Average number of EVs by changing the time between two successive reservation requests

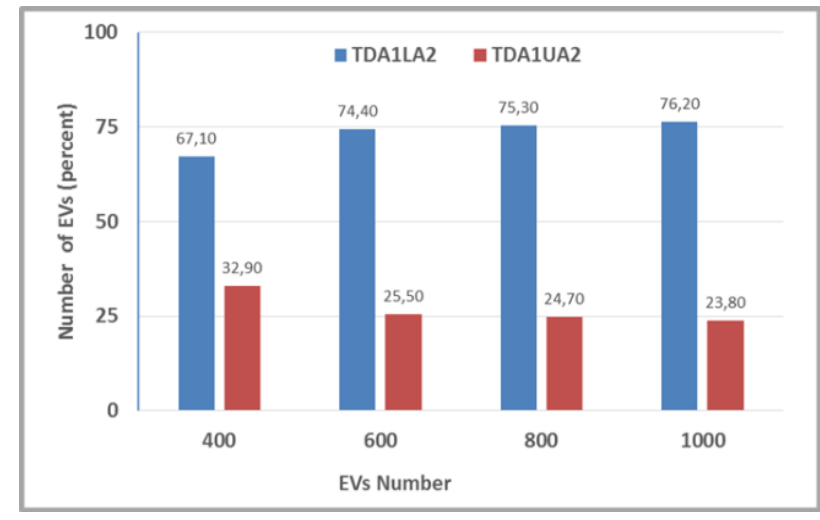

Figure 5. Average number of EVs by changing the EVs number that need charging 
Figure 6 shows a comparison between ALs 1 and ALs 2 at the mean of the travel time of all our EVs samples, the calculation of the average is opered at each instant ti, the arrival time of a new reservation request, which changes increasingly and we add to them an interval of time deltaT which is fixed at 100 seconds. Thus, we fixed the number of city EVs at 800 that will need charging. The results obtained from this comparison have shown that the average trip time estimated by Als 1 is all the time less than that estimated by Als 2 . Therefore, the Als 1 is more optimal compared to the ALs 2 and especially in the distribution of EVs, in a balanced way, on charging stations installed in the city.



Figure 6. Average trip duration

\section{CONCLUSION}

In this paper, we have proposed an architecture of a system that can effectively respond to EVs charging reservation requests in EVPSS. The decision to select an EVPSS, where the travel time of the EV driver to their destination is minimal, takes into account the EVs locally parking at EVPSS, the EVs performing remote charging reservations, arrival at the EVPSS, the expected charging time in EVPSS, and the travel time of the EVPSS to the destination. Thus, our proposed system sends an EVPSS change notification for electric vehicles moving towards its destination; due to a new reservation of an EV that will arrive before the EV having a reservation in the same EVPSS. The advantage of our proposed system, compared to what is proposed in the previous works, has been evaluated in the scenario of the Agadir city, in terms of minimizing the duration of the journey. The results showed the effectiveness of our proposal especially at peak periods and in the cities have a large number of EVs.

\section{REFERENCES}

[1] Highway Vehicles Responsible for a Declining Share of Pollutants, October 2017, [online] Available: https://energy.gov/eere/vehicles/articles/f act-998-october-9-2017-highway-vehicles-responsible-declining-share.

[2] J. L. Salmon, L. Willey, D. Vasquez and C. Christensen, "Systems analysis of EV adoption and criteria pollutant accumulation during inversion events," 2018 Annual IEEE International Systems Conference (Sys Con), Vancouver, BC, 2018, pp. 1-8.

[3] C. E. S. Thomas, "Transportation options in a carbon-constrained world: Hybrids, plug-in hybrids, biofuels, fuel cell electric vehicles, and battery electric vehicles," Int J Hydrogen Energy, vol. 34, no. 23, pp. 9279-9296, 2009.

[4] L. Dickerman and J. Harrison, "A New Car, a New Grid," in IEEE Power and Energy Magazine, vol. 8, no. 2, pp. 55-61, March-April 2010.

[5] A. G. Boulanger, A. C. Chu, S. Maxx and D. L. Waltz, "Vehicle Electrification: Status and Issues," in Proceedings of the IEEE, vol. 99, no. 6, pp. 1116-1138, June 2011.

[6] C. Thiel, J. Krause, and P. Dilara, "Electric vehicles in the eu from 2010 to 2014-is full scale commercialisation near," Luxembourg: Publications Office, pp. 1-40, 2015.

[7] J. K. Nor, "Art Of Charging Electric Vehicle Batteries," WESCON/'93. Conference Record, pp. 521-525, 28-30 Sep. 1993

[8] T. Winkler, P. Komarnicki, G. Mueller, G. Heideck, M. Heuer and Z. A. Styczynski, "Electric vehicle charging stations in Magdeburg," 2009 IEEE Vehicle Power and Propulsion Conference, Dearborn, MI, 2009, pp. 60-65.

[9] R. Navid, B. Kashanizadeh, M. Vakilian, and S. A. Barband, "Optimal placement of fast charging station in a typical microgrid in Iran," in Proc. IEEE Int. Conf. Eur. Energy Market(EEM), Stockholm, Sweden, 1-7, 2013

[10] X. Bi and W. K. S. Tang, "Logistical Planning for Electric Vehicles Under Time-Dependent Stochastic Traffic," in IEEE Transactions on Intelligent Transportation Systems, vol. 20, no. 10, pp. 3771-3781, Oct. 2019. 
[11] A. Bourass, S. Cherkaoui and L. Khoukhi, "Secure Optimal Itinerary Planning for Electric Vehicles in the Smart Grid," in IEEE Transactions on Industrial Informatics, vol. 13, no. 6, pp. 3236-3245, Dec. 2017.

[12] H. Qin and W. Zhang," Charging scheduling with minimal waiting in a network of electric vehicles and charging stations," in Proc. ACM Int.Workshop Veh. Inter-Netw., Las Vegas, NV, USA, pp. 51-60, Sep. 2011.

[13] Y. Luo, T. Zhu, S. Wan, S. Zhang, and K. Li, "Optimal charging scheduling for large-scale EV (electric vehicle) deployment based on the interaction of the smart-grid and intelligent-transport systems," Energy, vol. 97, pp. 359-368, Feb. 2016.

[14] S. Yang, "Price-responsive early charging control based on data mining for electric vehicle online scheduling," Electric Power Systems Research, vol. 167, pp. 113-121, 2019.

[15] Y. Cao, N. Wang, G. Kamel and Y. Kim, "An Electric Vehicle Charging Management Scheme Based on Publish/Subscribe Communication Framework," in IEEE Systems Journal, vol. 11, no. 3, pp. 1822-1835, Sept. 2017.

[16] Y. Cao et al., "Toward Efficient, Scalable, and Coordinated On-the-Move EV Charging Management," in IEEE Wireless Communications, vol. 24, no. 2, pp. 66-73, April 2017.

[17] M. Gharbaoui et al., "An advanced smart management system for electric vehicle recharge," in Proc. IEEE IEVC, Greenville, SC, USA, pp. 1-8, Mar. 2012.

[18] Y. Cao, X. Zhang, R. Wang, L. Peng, N. Aslam, and X. Chen, "Applying DTN routing for reservation-driven EV Charging management in smart cities," in Proc. IEEE International Wireless Communications and Mobile Computing (IWCMC), Valencia, Spain, pp. 1471-1476, June 2017.

[19] Y. Cao, T. Wang, O. Kaiwartya, G. Min, N. Ahmad and A. H. Abdullah, "An EV Charging Management System Concerning Drivers' Trip Duration and Mobility Uncertainty," in IEEE Transactions on Systems, Man, and Cybernetics: Systems, vol. 48, no. 4, pp. 596-607, April 2018.

[20] I. El-fedany, D. Kiouach and R. Alaoui, "Framework for optimizing the charging time of electric vehicles in public supply station deployed in smart cities," In Proc IEEE CiSt'18, Marrakesh, Morocco, pp. 537-541, Octobre, 2018.

[21] Y. Cao, et al., "Toward Anycasting-Driven Reservation System for Electric Vehicle Battery Switch Service," in IEEE Systems Journal, vol. 13, pp. 906-917, May 2018.

[22] Y. Cao et al., "A Cost-Efficient Communication Framework for Battery-Switch-Based Electric Vehicle Charging," in IEEE Communications Magazine, vol. 55, no. 5, pp. 162-169, May 2017.

[23] K. Sarrafan, K. M. Muttaqi, D. Sutanto and G. Town, "An intelligent driver alerting system for real-time range indicator embedded in electric vehicles," 2016 IEEE Industry Applications Society Annual Meeting, Portland, OR, 2016, pp. 1-7.

[24] B. Zhou, Q. Chen, P. Xiao and L. Zhao, "On the Spatial Error Propagation Characteristics of Cooperative Localization in Wireless Networks," in IEEE Transactions on Vehicular Technology, vol. 66, no. 2, pp. 1647-1658, Feb. 2017.

[25] B. Zhou, Q. Chen and P. Xiao, "The Error Propagation Analysis of the Received Signal Strength-Based Simultaneous Localization and Tracking in Wireless Sensor Networks," in IEEE Transactions on Information Theory, vol. 63, no. 6, pp. 3983-4007, June 2017.

[26] "Maps and map of Moroccan cities," [Online]. Available: http://www.mapsdumaroc.com/ 